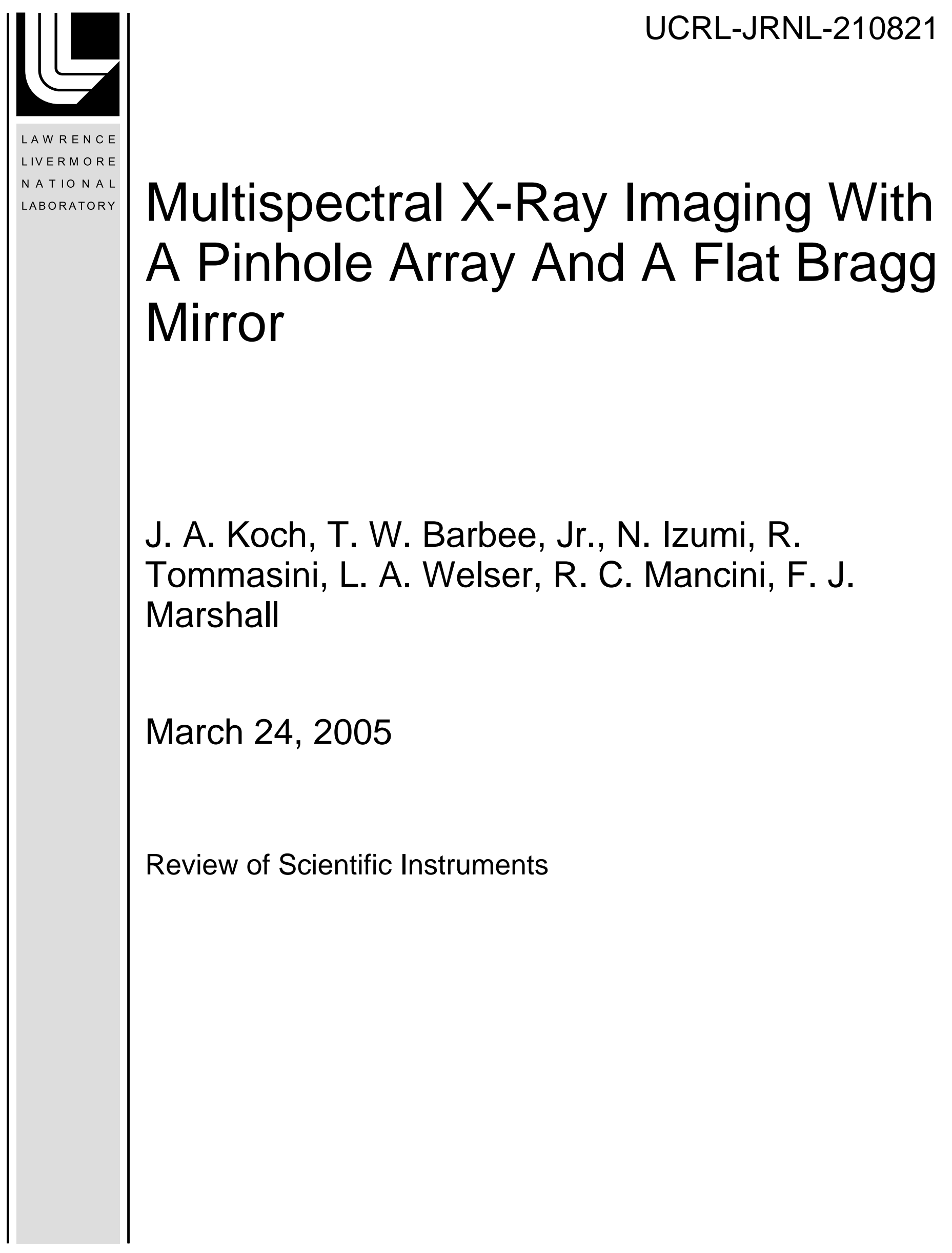


This document was prepared as an account of work sponsored by an agency of the United States Government. Neither the United States Government nor the University of California nor any of their employees, makes any warranty, express or implied, or assumes any legal liability or responsibility for the accuracy, completeness, or usefulness of any information, apparatus, product, or process disclosed, or represents that its use would not infringe privately owned rights. Reference herein to any specific commercial product, process, or service by trade name, trademark, manufacturer, or otherwise, does not necessarily constitute or imply its endorsement, recommendation, or favoring by the United States Government or the University of California. The views and opinions of authors expressed herein do not necessarily state or reflect those of the United States Government or the University of California, and shall not be used for advertising or product endorsement purposes. 


\title{
Multispectral X-ray Imaging with a Pinhole Array and a Flat Bragg Mirror
}

\author{
J. A. Koch, T. W. Barbee, Jr., N. Izumi, R. Tommasini \\ University of California, Lawrence Livermore National Laboratory \\ P.O. Box 808, L-481, Livermore CA, 94550 \\ L. A. Welser, R. C. Mancini \\ Department of Physics, University of Nevada, Reno NV \\ F. J. Marshall \\ Laboratory for Laser Energetics, University of Rochester, Rochester NY
}

\begin{abstract}
We describe a multiple monochromatic x-ray imager designed for implosion experiments. This instrument uses an array of pinholes in front of a flat multilayered Bragg mirror to provide many individual quasi-monochromatic $\mathrm{x}$ ray pinhole images spread over a wide spectral range. We discuss design constraints and optimizations, and we discuss the specific details of the instrument we have used to obtain temperature and density maps of implosion plasmas.
\end{abstract}




\section{Introduction}

Laser-produced plasmas are sufficiently hot to emit most of their electromagnetic radiation in the x-ray spectral region. For this reason, diagnosis of laserproduced plasma often relies on $\mathrm{x}$-ray spectroscopy and x-ray imaging. X-ray spectroscopy can be used as a diagnostic of plasma conditions through application of conventional line- and continuum-based measurements [1], and xray imaging can provide information on the structure of the plasma. These techniques have been applied since the early years of laser-produced plasma development.

A very powerful diagnostic can be realized by combining $x$-ray spectroscopy and $\mathrm{x}$-ray imaging. Multispectral x-ray imaging utilizes multiple quasimonochromatic $x$-ray images obtained over a spectral range encompassing important spectral features, such as emission lines that can be used as temperature and density diagnostics. Spatial localization of the quasimonochromatic $\mathrm{x}$-ray emission allows conventional spectroscopic techniques to be applied point by point in the plasma, providing local temperature and density information. When combined with time resolution by use of a gated x-ray imaging detector [2], multiple multispectral x-ray imaging views can provide detailed data on the time evolution of temperature and density maps within the plasma.

Multispectral x-ray imaging can be accomplished utilizing the properties of Bragg crystals. Spherically-bent [3] or toroidally-bent [4] Bragg crystals can be used as imaging elements, and an array of such crystals - tuned for different energies through slight variations in Bragg angles - can provide a number of quasi-monochromatic images at discrete energies across a desired range [5]. A simpler approach [6] uses an array of pinholes as the imaging element, and uses 
a flat Bragg mirror to provide energy dispersion. The latter approach can be less efficient [7], but is simpler and more powerful because a dense array of quasimonochromatic images can be obtained over a small numerical aperture simply by packing many pinholes into a substrate. The very large number of images allows for fine variations in the central x-ray energy of each image and allows many images with the same central x-ray energy to be superimposed, improving signal-to-noise $(\mathrm{S} / \mathrm{N})$ ratio. Decoupling imaging from dispersion also provides more flexibility in choosing the dispersion by varying the interplanar spacing of the Bragg mirror. Finally, a pinhole-based instrument is far simpler to design, characterize and align.

Starting with the concept of Yaakobi [6], we have optimized the geometry of a pinhole-based multiple monochromatic imager (MMI) for use in characterizing temperature and density profiles in inertial-confinement fusion (ICF) implosion plasmas [8-11]. In Section 2 we discuss the analytical considerations involved with specifying the design of such an instrument. In Section 3 we describe the instrument we have used to characterize ICF plasma temperature and density profiles, and in Section 4 we summarize our results and discuss future applications.

\section{Design Considerations}

The geometry of the MMI is shown in Figure 1. The source projects a shadow of the pinhole aperture onto a Bragg mirror, and the mirror reflects this shadow onto a detector that can be time-integrating or gated. The imaging properties can be understood by ignoring the mirror and following the dotted-line ray paths in Fig. 1. The mirror essentially serves to redirect the rays and restrict the spectral 
bandwidth of the reflected light by absorbing those x-ray wavelengths that do not satisfy the Bragg condition.

The detector spatial resolution and desired source resolution $\sigma$ set the required magnification $\mathrm{M}$, and the detector height $\mathrm{H}$ and distance $\mathrm{L}$ set the angular range of the pinhole array. The detector distance is typically fixed by other constraints, and the source diameter $\mathrm{F}$ together with the magnification set the pinhole spacing. Solving for the pinhole/target distance $\mathrm{p}$, pinhole diameter $\mathrm{D}$ (approximately including diffraction according to the x-ray wavelength $\lambda$ [7]), pinhole spacing 1 , and half-angle $\phi$ of the pinhole array, we have:

$$
\begin{aligned}
& p=L /(M+1) \\
& D^{2}=\frac{M^{2} \sigma^{2}}{2(M+1)^{2}}\left(1+\left(1-\left(\frac{4.88 \lambda L}{M \sigma^{2}}\right)^{2}\right)^{1 / 2}\right) \\
& l=\frac{F(M+1)}{M} \\
& \phi=\arctan \left(\frac{H}{2 L}\right)
\end{aligned}
$$

The central Bragg angle $\theta$ and mirror interplanar spacing $\mathrm{d}$ determine the wavelength range covered by the pinhole array. From Fig. 1,

$$
\begin{aligned}
& \lambda_{2}=2 d \sin (\theta+\phi) \\
& \lambda_{1}=2 d \sin (\theta-\phi)
\end{aligned}
$$




$$
\frac{\Delta \lambda}{\lambda}=\frac{\lambda_{2}-\lambda_{1}}{\lambda_{0}}=\frac{2 \sin \phi}{\tan \theta}
$$

Equation (7) shows that the relative bandwidth covered by the detector scales inversely with $\tan \theta$ and therefore approximately scales with the interplanar spacing. The spectral width covered by a single image is approximately $\Delta \lambda \mathrm{MF} / \mathrm{H}$, or

$$
\Delta \lambda_{\text {image }}=\frac{4 d \cos \theta \sin \phi M F}{H}=\frac{4 d \cos \theta M F}{\sqrt{4 L^{2}+H^{2}}} \approx \frac{2 d M F}{L}
$$

which shows that larger interplanar spacings increase the spectral content of each image proportional to $\mathrm{d}$. Greater monochromaticity requires smaller interplanar spacings.

For a mirror-target distance $\mathrm{u}$, the required mirror length $\mathrm{S}$ is:

$$
S=u \sin \phi\left(\frac{1}{\sin (\theta+\phi)}+\frac{1}{\sin (\theta-\phi)}\right)
$$

Typically $S$ is determined by available commercial mirror substrate lengths and $\theta$ is determined by the desired spectral coverage, implying that $\phi$ and therefore $\mathrm{H}$ are determined.

The pinhole array can be rotated about the optical axis in order to provide spectral coverage equally at all wavelengths within the total spectral range [6]. For a detector width $\mathrm{K}$, the number of images that can be obtained in a row is approximately K/MF. If the pinholes are packed in a square array, then the optimum tilt angle $\chi$ is: 


$$
\chi=\arctan \left(\frac{M F}{K}\right)
$$

Finally, for throughput, the number of photons per resolution element is maximized by placing the pinhole as close as possible to the target [7]. This configuration also maximizes spatial resolution for a given pinhole aperture by minimizing diffraction. Ignoring diffraction, the number of photons per resolution element $\mathrm{N}$ can be written as

$$
N=I_{0} \frac{\pi^{2}(M+1)^{2} D^{4}}{16 M^{2} p^{2}} \eta \approx 0.6 I_{0} \frac{D^{4}}{p^{2}} \eta
$$

where the approximation holds for large $\mathrm{M}, \mathrm{I}_{0}$ is the source emissivity in photons/unit area/steradian and $\eta$ is the Bragg mirror-limited efficiency. The pinhole diameter is determined by spatial resolution requirements, so moving the pinhole closer to the target improves photon statistics at constant spatial resolution. The Bragg mirror efficiency depends on the pinhole aperture and distance and on the relative emission spectrum and mirror bandwidths, and can be estimated for various cases [7].

\section{MMI Instrument Design and Experimental Data}

The instrument described here was designed for indirect-drive implosions experiments at the Omega Laser Facility [12]. In these experiments, a plastic capsule containing Ar-doped deuterium gas is imploded by x-rays generated by laser interactions with a gold radiation cavity [13]. At times near peak compression, electron densities exceed $10^{24} \mathrm{~cm}^{-3}$ and electron temperatures exceed $1 \mathrm{keV}$, so that the Ar dopant emits hydrogen-like and helium-like 
resonance lines that can be used as temperature and density diagnostics. The instrument was designed to provide multiple quasi-monochromatic images across the $\sim 3-5 \mathrm{keV}$ spectral region covering these emission lines, so that maps of temperature and density can be obtained by Abel inversion and either analytical manipulation [8] or search and reconstruction techniques [10, 11]. We designed the instrument to have high sensitivity by moving the pinhole and detector in as close to the target as possible, limited by interference with the laser beams used to drive the implosion. Sensitivity, spectral coverage and image quality requirements constrained us to use a multilayer Bragg mirror as the dispersion element. These mirrors are efficient, relatively broad-band, and extremely flat; however, layer deposition technology limitations preclude interlayer spacings smaller than $\sim 1.5 \mathrm{~nm}$, requiring use at grazing $\left(\sim 6^{\circ}\right)$ angles of incidence. Suitable high-quality crystals, such as $\mathrm{Si}$ or Ge, have much smaller bandwidths and correspondingly small efficiencies. An earlier version of the instrument utilized a graphite crystal, and while this instrument did provide high efficiency due to the broad mosaic bandwidth of the crystal, the images were too distorted to be useful.

The instrument geometry is shown schematically in Fig. 2. The target-pinhole distance $p=15.9 \mathrm{~mm}$ and the target-detector distance $L=143 \mathrm{~mm}$, giving a magnification of 8 . The pinhole array has $12805 \mu \mathrm{m}$-diameter square-packed pinholes in a $1 \times 5.5 \mathrm{~mm}$ region of the $25 \mu \mathrm{m}$-thick Ta substrate, and is mounted onto the target. The pattern is rotated $7.125^{\circ}$ about the optical axis, and overfills the detector area in order to allow for tolerance in positioning. The Bragg mirror is a $\mathrm{W} / \mathrm{B}_{4} \mathrm{C}$ multilayer on a $50.8 \mathrm{~mm}$-diameter superpolished substrate with an interplanar spacing of approximately $1.51 \mathrm{~nm}$, and is placed 82 $\mathrm{mm}$ from the target. Internal baffles restrict the spectral coverage to $3-4.4 \mathrm{keV}$ 
with a spectral resolution of approximately $\Delta \mathrm{E} / \mathrm{E} \approx 0.5 \%$. This spectral range projects to a length $\mathrm{H} \approx 6.5 \mathrm{~mm}$ at the detector plane.

The detector is a time-integrated charge injection device (CID) x-ray detector with a pixel size of $40 \mu \mathrm{m}$ and an image area of $24 \times 36 \mathrm{~mm}$ [14]. This detector is convenient, sensitive, and relatively large, but it does not provide time resolution. We have also used the MMI, with slight modifications to the instrument geometry and with a hexagonally-packed pinhole array, with a gated $\mathrm{x}$-ray framing camera. This detector provides four individually-timed strips that are $5.7 \mathrm{~mm}$ wide and separated by $3.25 \mathrm{~mm}$. Typical data from both detectors are shown in Fig. 2.

A key feature of the MMI data is that multiple images across a band of x-ray wavelengths can be numerically added to improve signal-to-noise ratio. The individual pinhole images are generally asymmetric because spatial information is coupled with spectral information, but the far wings of the profiles are generally round, so that contours of low constant relative brightness can be used to locate the centroids of each individual image. Alternatively, the entire pattern of images can be fit to a regular pattern determined by the known properties of the pinhole array coupled with undetermined constants to characterize possible tips and tilts of the array with respect to the optical axis. Both approaches have been shown to provide good results [8-11].

\section{Discussion and Conclusions}

We have described a multispectral $x$-ray imager that provides a large number of quasi-monochromatic individual pinhole images spread over a wide spectral range. The instrument design parameters were optimized for specific implosion 
experiments at the Omega Laser Facility, but we have discussed various design constraints that allow different optimizations for other applications.

The main advantages of this technique compared with other options, including arrays of bent crystals, are: simplicity of fabrication, characterization and alignment; relatively high achievable spatial resolution $(\sim 5 \mu \mathrm{m})$; the ability to provide a very large number of images that can be compared and numerically added to improve signal-to-noise ratio; and the ability to provide many images across a chosen spectral range with a narrow separation of central wavelengths. The main disadvantage is achievable image brightness, due to pinhole imaging constraints compounded by spectral dispersion from the Bragg mirror. This constraint becomes particularly severe when natural crystals are utilized, generally requiring the use of multilayer mirrors that are not capable of providing spectral monochromaticity better than $\sim 0.5 \%$.

The instrument described here has been utilized to provide temperature and density maps of Ar-doped implosion cores by multispectral x-ray imaging across the spectral region containing Ar resonance line emission. A similar approach would be well suited for viewing continuum emission images from undoped cores [15].

Finally, we point out that the technique described here can be applied to other experiments. For example, gated multispectral x-ray images could provide useful information in colliding plasma and turbulent mix experiments, where different materials emitting different spectra might be spatially localized.

\section{Acknowledgments}

This work was performed under the auspices of the U.S. Department of Energy by the University of California Lawrence Livermore National Laboratory under 
contract No. W-7405-ENG-48, and by the University of Nevada, Reno, under DOE NLUF Grant No. DE-FG03-03SF22696. We gratefully acknowledge S. Dalhed, C. Damian, V. Glebov, I. Golovkin, S. Haan, B. Hammel, L. Klein, O. Landen, R. Lee, M. Marinak, D. Meyerhofer, H. Nishimura, Y. Ochi, S. Regan, V. Rekow, T. Sangster, V. Smalyuk, C. Sorce, J. Soures, R. Turner, R. Wallace and the staff at LLE for discussions and support. 


\section{References}

[1] H. R. Griem, Principles of Plasma Spectroscopy (Cambridge University Press, Cambridge, 1997).

[2] J. D. Kilkenny, Laser and Particle Beams 9, 49 (1991).

[3] Y. Aglitskiy, T. Lehecka, S. Obenschain, S. Bodner, C. Pawley, K. Gerber, J. Sethian, C. M. Brown, J. Seely, U. Feldman, G. Holland, Appl. Opt. 37, 5253 (1998) and references therein.

[4] E. Förster, K. Gäbel, I. Uschmann, Laser and Particle Beams 9, 135 (1991).

[5] I. Uschmann, K. Fujita, I. Niki, R. Butzbach, H. Nishimura, J. Funakura, M. Nakai, E. Förster, K. Mima, Appl. Opt. 39, 5865 (2000)

[6] B. Yaakobi, F. J. Marshall, D. K. Bradley, Appl. Opt. 37, 8074 (1998).

[7] J. A. Koch, O. L. Landen, T. W. Barbee, P. Celliers, L. B. Da Silva, S. G. Glendinning, B. A. Hammel, D. H. Kalantar, C. Brown, J. Seely, G. R. Bennett, W. Hsing, Appl. Opt. 37, 1784 (1998).

[8] J. A. Koch, N. Izumi, L. A. Welser, R. C. Mancini, S. W. Haan, T. W. Barbee, Jr., S. Dalhed, I. E. Golovkin, L. Klein, R. W. Lee, F. J. Marshall, D. Meyerhofer, H. Nishimura, Y. Ochi, T. C. Sangster, V. Smalyuk (submitted for publication).

[9] J. A. Koch, T. W. Barbee, Jr., S. Dalhed, S. Haan, N. Izumi, R. W. Lee, L. A. Welser, R. C. Mancini, F. J. Marshall, T. C. Sangster, V. A. Smalyuk, J. M. Soures, L. Klein, in Inertial Fusion Sciences and Applications 2003 (American Nuclear Society, La Grange, 2004), p. 857.

[10] L. A. Welser, R. C. Mancini, J. A. Koch, N. Izumi, H. Dalhed, H. Scott, T. W. Barbee, Jr., R.W. Lee, I. E. Golovkin, F. Marshall, J. Delettrez, L. Klein, J. Quant. Spectrosc. Radiat. Transfer 81, 487 (2003). 
[11] L. A. Welser, R. C. Mancini, J. A. Koch, S. Dalhed, R. W. Lee, I. E. Golovkin, F. Marshall, J. Delettrez, L. Klein, Rev. Sci. Instrum. 74, 1951 (2003).

[12] T. R. Boehly, D. L. Brown, R. S. Craxton, R. L. Keck, J. P. Knauer, J. H. Kelly, T. J. Kessler, S. A. Kumpan, S. J. Loucks, S. A. Letzring, F. J. Marshall, R. L. McCrory, S. F. B. Morse, W. Seka, J. M. Soures, C. P. Verdon, Opt. Commun. 133, 495 (1997).

[13] J. D. Lindl, Inertial Confinement Fusion (AIP Press, New York, 1998) and references therein.

[14] F. J. Marshall, T. Ohki, D. McInnis, Z. Ninkov, J. Carbone, Rev. Sci. Instrum. 72, 713 (2001).

[15] J. A. Koch, S. W. Haan, R. C. Mancini, J. Quant. Spectrosc. Radiat. Transfer 88, $433(2004)$. 


\section{Figure Captions}

Figure 1: Sketch of the basic instrument geometry from the side and top.

Figure 2: Schematic of the MMI operational geometry and representative data from a time-integrated CID detector (left) and a gated framing camera (right). Emission bands corresponding to Ar He- $\alpha$, Ly- $\alpha$, He- $\beta$ and Ly- $\beta$ are shown along with a Ti K- $\alpha$ absorption band (visible in the left image only) caused by Ti doped into the shell. 


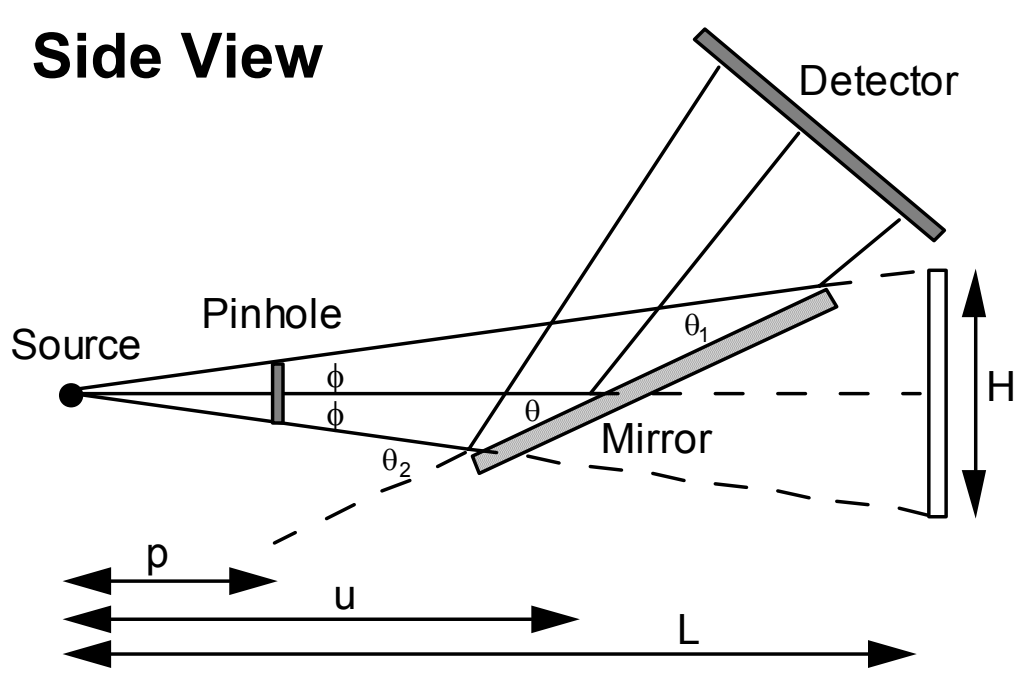

Top View

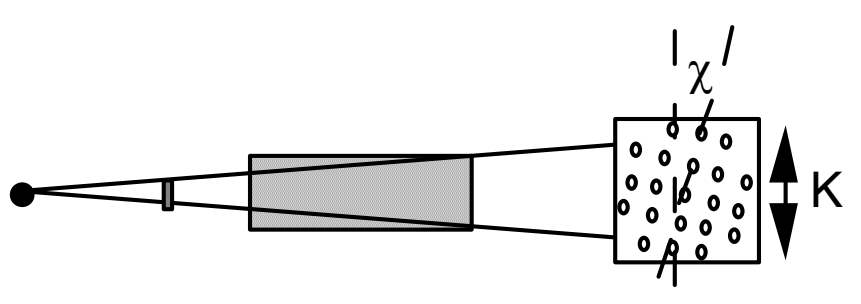

Figure 1 


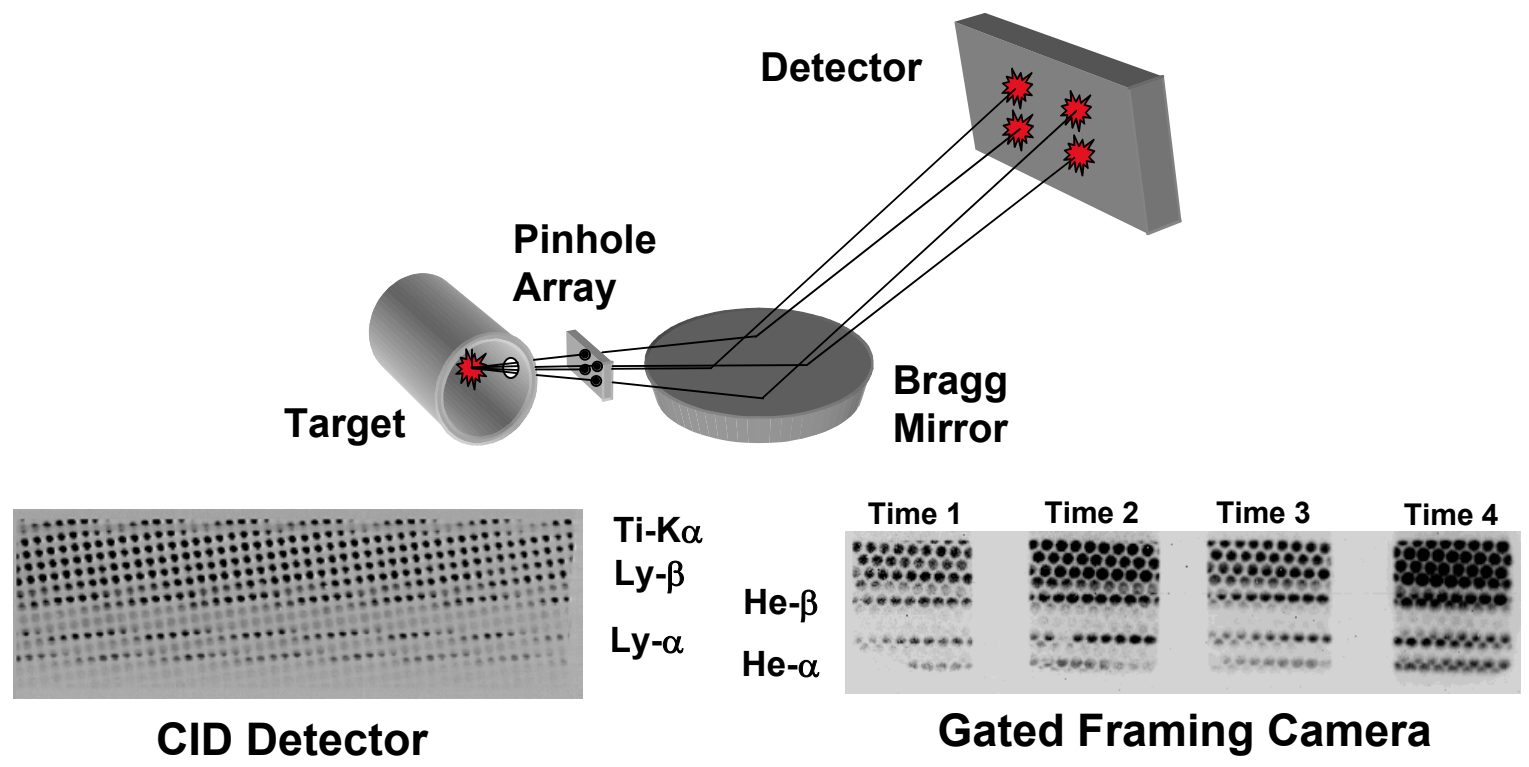

Figure 2 